\title{
Improved method for immunoperoxidase detection of membrane antigens in frozen sections
}

\author{
M A GREEN, L SVILAND, A J MALCOLM, A D J PEARSON* Departments of Pathology \\ and ${ }^{*}$ Child Health, University of Newcastle upon Tyne
}

SUMMARY The visualisation of membrane antigens in frozen sections using metal-enhanced 3,3'diaminobenzidine (DAB) was investigated. Particular attention was paid to the degree of reaction enhancement, the disruptive effect on morphological detail, and the ease of the techniques. The best result was obtained using nickel-modified DAB at pH 6.0 to develop the peroxidase enzyme, with further enhancement in cobalt chloride at neutral pH. Silver methenamine enhancement is also possible but can give rise to non-specific or background staining.

Immunoperoxidase is the most widely used method to detect antigens in tissue, and 3,3'diaminobenzidine (DAB) is the most common substrate for the visualisation of the enzyme. Attempts have been made to improve the sensitivity of the detection and the intensity of the reaction. Alternatives to DAB, though safer to handle, have not been found to be as good.' Most studies, therefore, have tried to improve the DAB reaction in paraffin wax sections, ${ }^{23}$ plastic embedded tissue, ${ }^{4}$ or a model system, such as nitrocellulose strips. ${ }^{1}$ These techniques have entailed either modifying the DAB solution by the addition of heavy metal ions to produce a more dense reaction, or by enhancement procedures which exploit the ability of polymerised DAB to reduce metal ions.

Some techniques require the use of unpleasant or dangerous chemicals ${ }^{5}$ or are too time consuming to be useful in a routine laboratory ${ }^{6}$; others produce an increase in intensity (density of the reaction product) but no improvement in sensitivity (total number of cells with visible reaction product). As many important antigens can be detected only in frozen tissue, an effective DAB enhancement procedure is needed for use in cryostat sections.

In this study techniques shown to produce good results in fixed tissue were developed for the demonstration of membrane antigens in cryostat sections and then compared for improved sensitivity, degree of nuclear preservation, the ease of the procedure and their applicability to the routine laboratory.

Accepted for publication 16 February 1989

\section{Material and methods}

Sections of tonsil $(4 \mu \mathrm{m})$ were cut on a disposable blade to reduce cutting artefacts, air dried overnight at room temperature, and fixed in acetone for 10 minutes at room temperature before being subjected to the primary and secondary antibody stages of a standard indirect immunoperoxidase technique ${ }^{7}$ for OKT6 (CD1), LeuM2 (monocytes/macrophages), and TA-1 (T-cell activation marker). These antibodies were chosen for their variable staining of different types of cell (OKT6), weak staining (TA-1), and for the low number of positive cells in tonsil (LeuM2). The standard DAB procedure with imidazole was compared with the other techniques. Variations in results were minimised by using the same batch of DAB throughout (Isopac from Sigma), by cutting all frozen sections at the same thickness, and by keeping incubation times and antibody dilutions constant. The sections then received the following peroxidase development methods:

(a) Standard DAB (sDAB): $50 \mathrm{mg} \%$ DAB (Sigma) in Tris-buffered saline pH 7.6 (TBS) and $0.068 \%$ imidazole (Sigma). ${ }^{8}$ Sections were incubated for the optimum time, determined microscopically.

(b) Heavy metal modification of $D A B$ using

(i) Copper and cobalt ${ }^{2}: 2 \mathrm{ml}$ of a $1 \%$ solution of cobalt chloride or copper sulphate in Tris buffer at neutral $\mathrm{pH}$ was added to $100 \mathrm{ml}$ of sDAB. The sections were incubated in both metal-DAB solutions for five minutes before hydrogen peroxide was added to a final concentration of $0.01 \%$ and the reaction completed.

(ii) $\mathrm{Nickel}^{19}$ : the solution comprises $1 \%$ nickel 
sulphate, $0.068 \%$ imidazole, and $0.8 \%$ sodium chloride in $0.1 \mathrm{M}$ acetate buffer, $\mathrm{pH} 6 \cdot 0$. Hydrogen peroxide is added to a final concentration of $0.01 \%$ and the sections incubated until the reaction is complete.

(c) Post-DAB enhancement with

(i) Heavy metals: after sDAB development the sections were washed briefly in TBS then immersed in $0.5 \%$ solutions of cobalt chloride, nickel sulphate, or

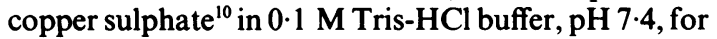
four minutes at room temperature.

(ii) Ferric-ferricyanide ${ }^{3}$ : after $\mathrm{sDAB}$ development sections were incubated at room temperature in a fresh mixture of three parts $1 \%$ ferric chloride to one part $0.1 \%$ potassium ferricyanide until background was observable microscopically (about 10 minutes).

(iii) Silver methenamine with and without subsequent gold toning ${ }^{4}$ : after the $\mathrm{SDAB}$ stage sections were washed well in distilled water and then incubated for 10 minutes in a mixture of 20 parts $3 \%$ hexamine to one part $5 \%$ silver nitrate to two parts $2.5 \%$ sodium tetraborate, preheated to $60^{\circ} \mathrm{C}$. After washing in distilled water $0.05 \%$ gold chloride toning was applied to some sections until the sections turned grey (about 20 seconds).

(iv) Silver intensification of DAB-reduced gold chloride: after SDAB development sections were immersed in $0.1 \%$ gold chloride for four minutes, washed well in distilled water, and then subjected to the silver methenamine method outlined in (iii).

(v) The Gallyas silver method ${ }^{6}$ : after the sDAB stage the sections were washed well in distilled water and then immersed in a mixture of equal parts solution $A$ (5\% anhydrous sodium carbonate) and solution $\mathbf{B}$ $(0.2 \%$ ammonium nitrate, $0.2 \%$ silver nitrate, $1 \%$ tungstosilicic acid and $0.5 \%$ formaldehyde) for 10 minutes.

After the above procedures the sections were washed in distilled water and counterstained with
Mayer's haematoxylin for brown reaction products, and nuclear fast red for black or green-blue results. After alcohol dehydration and clearing in xylene the sections were mounted in DPX resin.

(d) Hybrid DAB enhancement Sections were cut as above and reacted with the antibodies OKT6 and TA1, with a "no primary antibody" negative control. After peroxidase labelling, adjacent sections were developed with nickel-modified DAB and SDAB for comparison and then subjected to the following enhancements: (i) silver methenamine (SM) only; (ii) $0.5 \%$ cobalt chloride ( $\mathrm{Co}$ ) only; (iii) Co followed by SM; (iv) gold chloride followed by SM, and; (v) Co followed by gold followed by SM.

Gold toning was applied where necessary to remove background colouration.

A summary of methods tested is found in table 1 .

\section{Results}

Heavy metal modification of the $D A B$ produced a range of results, copper having little effect on intensity or sensitivity of the reaction; nickel giving a useful improvement in both areas. Nickel was also the simplest procedure of the three as the solution can be made up in large quantities, divided into aliquots, and stored at $-20^{\circ} \mathrm{C}$ for months. The cobalt-DAB solution auto-oxidises even at $-20^{\circ} \mathrm{C}$. All three metals gave adequate nuclear morphology and were simple to use.

Post DAB enhancement with

Copper, cobalt, and nickel produced a noticeable improvement in the nuclear preservation, with nucleoli and chromatin patterns much more obvious, especially after the cobalt treatment. The colour of the DAB was slightly darkened by the metals but no increase in sensitivity was observed. This step only adds a few minutes to the technique and the cobalt solution can be re-used.

Table 1 Summary of methodologies

\begin{tabular}{|c|c|c|c|c|}
\hline $\begin{array}{l}\text { Modification of } \\
\text { DAB with: }\end{array}$ & Buffer & Post $-D A B$ enhancement & Buffer & Subsequent steps \\
\hline Cobalt chloride & Tris & - & - & - \\
\hline Copper sulphate & Tris & - & - & - \\
\hline Nickel sulphate & Acetate & $\overline{C a}$ & $\bar{T}$. & - \\
\hline - & - & $\mathrm{CoC} 12$ & Tris & - \\
\hline - & - & $\mathrm{CuSO} 4$ & Tris & - \\
\hline - & - & $\mathrm{NiSO4}$ & Tris & - \\
\hline - & - & Ferric-ferricyanide & - & - \\
\hline - & - & Silver methenamine & - & $\overline{0 .}$ \\
\hline- & - & Gold chloride & - & Silver methenamine \\
\hline$\overline{-1}$ & - & Gallyas silver solution & - & - \\
\hline NiSO4 & Acetate & Silver methenamine & - & - \\
\hline NiSO4 & Acetate & $\mathrm{CoC} 12$ & Tris & $\bar{\sigma}-1$. \\
\hline NiSO4 & Acetate & $\mathrm{CoCl} 2$ & Tris & Silver methenamine \\
\hline $\mathrm{NiSO} 4$ & Acetate & Gold chloride & - & Silver methenamine \\
\hline NiSO4 & Acetate & $\mathrm{CoCl} 2$ & Tris & Gold chloride then silver methenamine \\
\hline
\end{tabular}



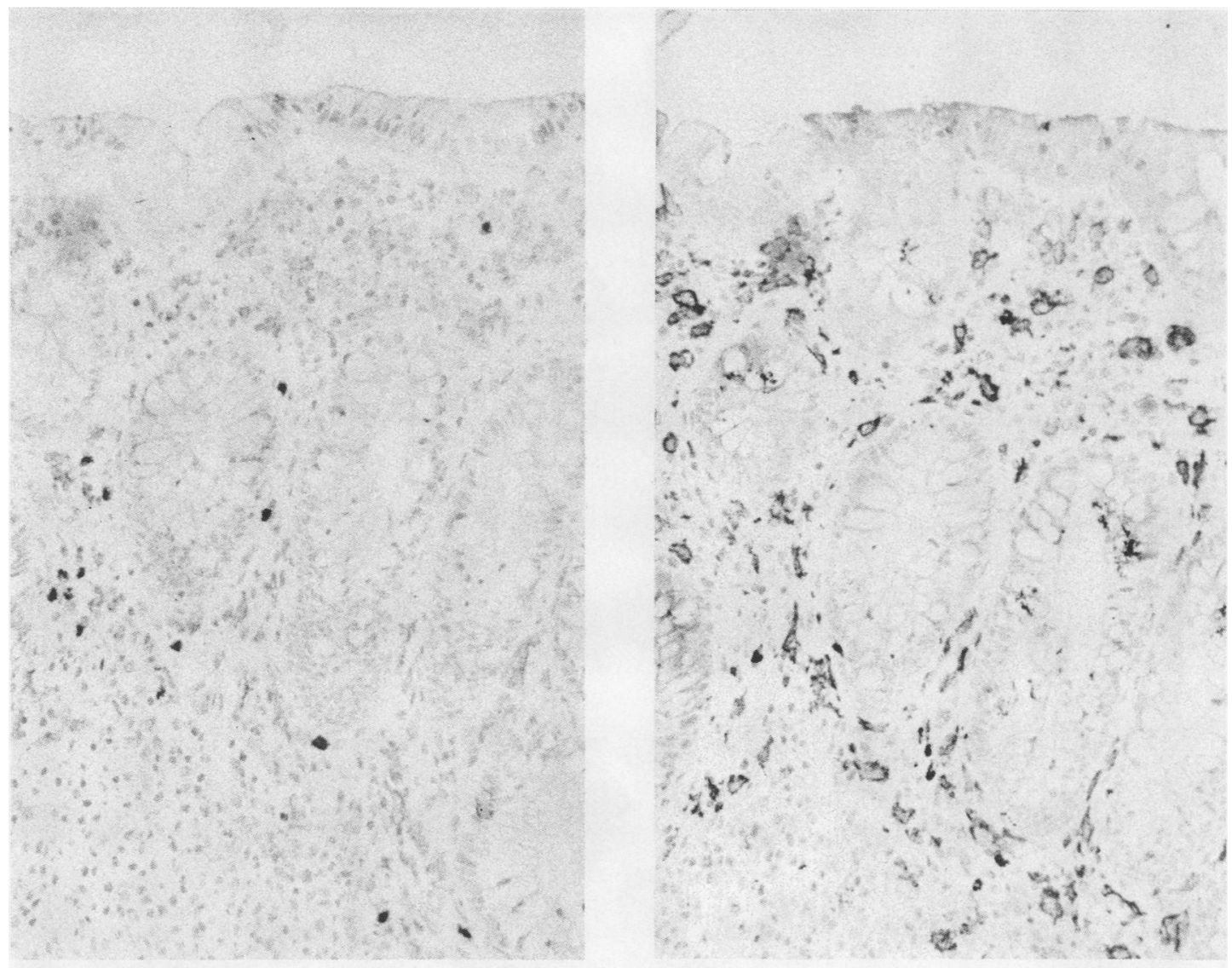

2

Figs 1 and 2 These sections show the increase in sensitivity of the NiDABCo methods. The same area of adjacent frozen sections of rectum was stained for RFD7 (macrophage marker), and developed with SDAB in 1 and NiDABCo in 2.

Ferric-ferricyanide treatment caused a colour change from brown to blue-green with a concurrent increase in the intensity of the reaction. This did not improve the sensitivity of the technique, however, and the morphological detail was seriously compromised.

Silver methenamine intensification produced a noticeable increase in both the intensity and the sensitivity of the technique. Cells not visible by $\mathrm{SDAB}$ were light brown after silver enhancement. Unfortunately, the morphological detail was again poor.

The binding of gold to the polymerised DAB produces nucleation sites for the precipitation of silver from subsequent solution. The result is a black reaction product and increased sensitivity. In common with other metal solutions, the gold chloride conferred some protection to the nuclei, which are adequately

Table 2 Summary of useful methods

\begin{tabular}{|c|c|c|c|}
\hline Method & Nuclear morphology & Reaction product & Comments \\
\hline $\begin{array}{l}\text { SDAB } \\
\text { DAB-Co }\end{array}$ & $\begin{array}{l}\text { Adequate } \\
\text { Very good nucleoli and chromatin } \\
\text { patterns }\end{array}$ & $\begin{array}{l}\text { Brown } \\
\text { Dark brown }\end{array}$ & $\overline{\text { Tris-cobalt can be re-used }}$ \\
\hline NiDABCo & Very good & Intense, black, crisp & $\begin{array}{l}\text { Easy to perform. Solution keeps well at }-20^{\circ} \mathrm{C} \text {. } \\
\text { Clean staining }\end{array}$ \\
\hline $\begin{array}{l}\text { NiDABCo-SM } \\
\text { sDABCo-SM }\end{array}$ & $\begin{array}{l}\text { Adequate } \\
\text { Good }\end{array}$ & $\begin{array}{l}\text { Intense, black } \\
\text { Intense, dark brown }\end{array}$ & $\begin{array}{l}\text { Lengthy technique, background problems } \\
\text { Good alternative, background less obvious }\end{array}$ \\
\hline
\end{tabular}



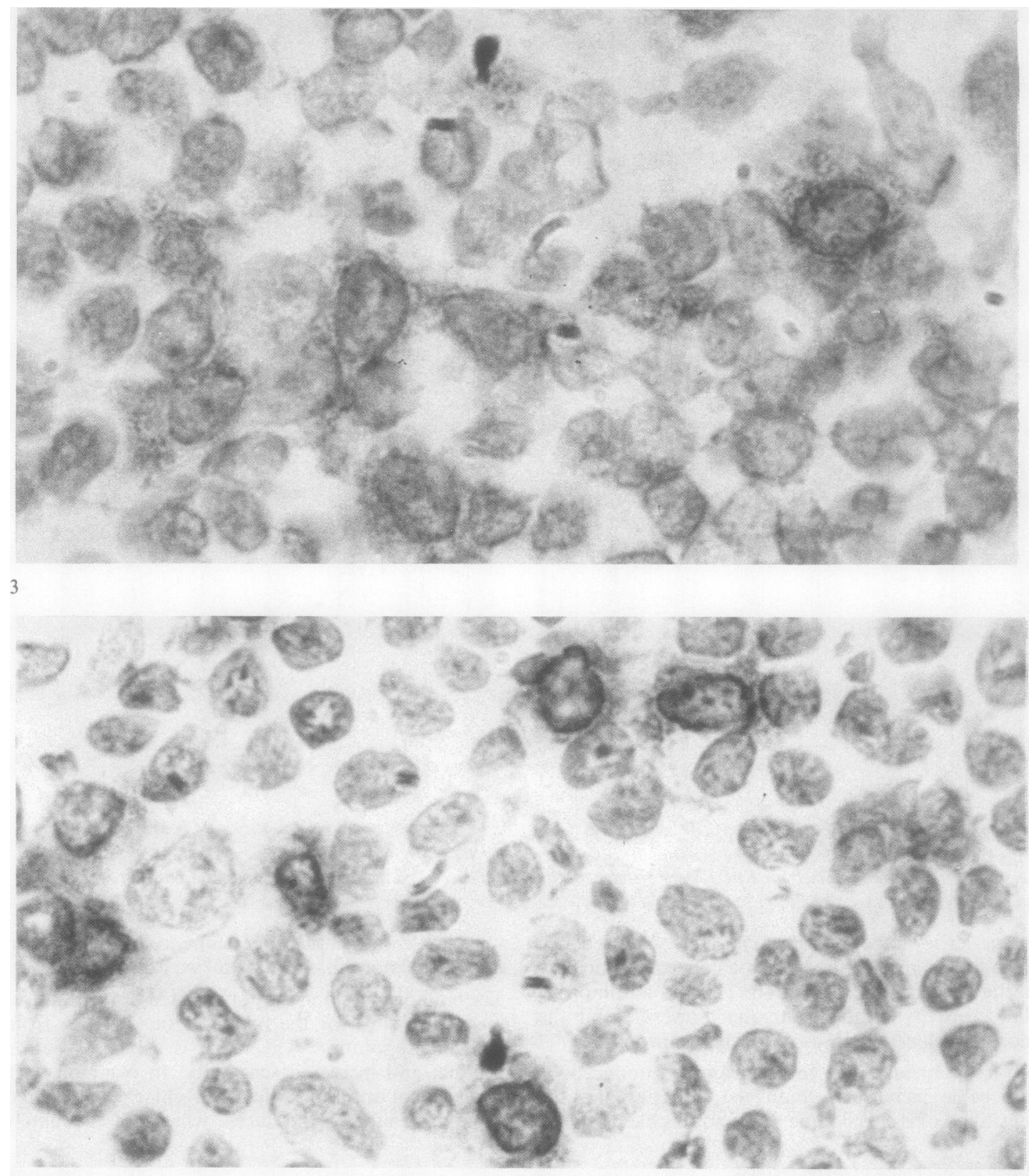

4

Figs 3 and 4 These sections show the effect of the cobalt chloride on the nuclear preservation. Adjacent frozen sections of tonsil were stained for Leu4 (Pan T cells) and developed with SDAB in 3 and sDABCo in 4.

preserved in this method. Gold toning, if used, should be as brief as possible to avoid replacement of the silver with gold.

The Gallyas method, although simple, did not give good results, with poor morphological detail and only slight intensification of the DAB. The method followed here omitted the four hour incubation step in thioglycollic acid to reduce background argyrophilia. 
Table 3 Comparison of cell counts with standard DAB and nickel modified DAB with cobalt enhancement

\begin{tabular}{|c|c|c|c|c|c|c|c|c|}
\hline & \multicolumn{4}{|l|}{$s D A B$} & \multicolumn{4}{|c|}{$N i D A B C o$} \\
\hline & OKT8 & $K i 67$ & Leu7 & $T A-1$ & $O K T 8$ & Ki67 & Leu7 & $T A-1$ \\
\hline $\begin{array}{l}\text { Rectum } \\
\text { Heart } \\
\text { Kidney } \\
\text { Liver } \\
\text { Tonsil }\end{array}$ & $\begin{array}{l}- \\
+ \\
+ \\
+\end{array}$ & $\begin{array}{l}- \\
+ \\
++ \\
+ \\
-\end{array}$ & $\begin{array}{l}+ \\
- \\
+ \\
- \\
+++\end{array}$ & $\begin{array}{l}+ \\
- \\
- \\
+\end{array}$ & $\begin{array}{l}- \\
++ \\
- \\
+++ \\
-\end{array}$ & $\begin{array}{l}- \\
+ \\
+++ \\
++ \\
-\end{array}$ & $\begin{array}{l}++ \\
\bar{t}+ \\
\bar{t}++\end{array}$ & $\begin{array}{l}+ \\
- \\
- \\
+ \\
++\end{array}$ \\
\hline
\end{tabular}

$+=0$ to 20 cells; $++=20$ to 100 cells; $+++=100$ or more cells.

The full method, and the fact that Gallyas recommends mounting in Canada Balsam, make this technique less applicable to the routine laboratory.

\section{Hybrid DAB enhancement}

In all cases $\mathrm{SDAB}$ resulted in a dark brown reaction product and nickel-DAB (NiDAB) black. NiDAB was always more sensitive than $\mathrm{SDAB}$, and where cobalt was used, the outlines of the nuclei were improved, although it did not protect fully against the effects of the silver methenamine solution. The silver intensification produced noticeably more background, a more intense reaction, but no increase in sensitivity, while gold-silver gave the worst background to the point of obscuring the positivity.

A summary of useful methods is shown in table 2 .

The best result was produced using NiDAB followed by Tris/cobalt enhancement (NiDABCo) as follows:

\section{Recommended method:}

(1) Thaw aliquot of nickel-DAB, add hydrogen peroxide to a concentration of $0.01 \%$, and filter.

(2) Wash sections in $0.1 \mathrm{M}$ acetate buffer ( $\mathrm{pH}$ 6) while the solution is filtering.

(3) Tip off excess buffer and incubate sections in Nickel-DAB $(95 \mathrm{mg}$ DAB $+1.6 \mathrm{~g} \mathrm{NaCl}+0.136 \mathrm{~g}$ imidazole $+2 \mathrm{~g} \mathrm{NiSO} 4+195 \mathrm{ml} \mathrm{0.1} \mathrm{M}$ acetate buffer, $\mathrm{pH}$ adjusted to $6 \cdot 0$, and made up to $200 \mathrm{ml}$ with buffer), until the reaction is complete and background is minimal, three to five minutes.

(4) Tip off substrate and wash briefly in Tris buffered saline (pH 7.6) twice for five seconds each time.

(5) Incubate in Tris/cobalt solution (1.2 Tris $+1.0 \mathrm{~g}$ $\mathrm{CoCl} 2+180 \mathrm{ml}$ distilled water, $\mathrm{pH}$ adjusted to $7 \cdot 2$ with $0 \cdot 1 \mathrm{M} \mathrm{HCl}$ and made up to $200 \mathrm{ml}$ ), for four minutes at room temperature. This solution can be reused.

(6) Wash briefly in distilled water for five seconds.

(7) Counterstain in $0.1 \%$ nuclear fast red in $5 \%$ aluminium sulphate, for two minutes.

(8) Wash in running tap water to remove traces of DAB and excess stain.

(9) Dehydrate in graded alcohols, clear in xylene, and mount in DPX.

\section{Comparison of NiDABCo with $s D A B$}

To confirm that the NiDABCo procedure was the optimal method tested, a variety of antigens were visualised in a range of frozen surgical and necropsy tissues. For each tissue and antibody combination adjacent sections were stained with NiDABCo and sDAB. The number of positive cells in equal areas of the adjacent sections was counted by two independent assessors.

The NiDABCo sections showed the same level of background staining as the sDAB but with a higher staining intensity, which meant that positive cells could be identified with more confidence. Nuclear preservation was also improved, again aiding cell identification.

The NiDABCo method was particularly useful for detecting the nuclear antigen recognised by $\mathrm{Ki67}$-for example, in kidney, the resulting black nuclei contrasting strongly with the red, negative nuclei. Black, cALLa positive cells were also more easily seen in a section of spleen containing a considerable amount of iron pigment. Occasional cells, such as lymphocytes in heart, stand out much more clearly, and this is an obvious advantage when searching for infiltrates. Table 3 shows the results of a representative selection of cell count comparisons.

\section{Discussion}

This study has shown that although previously published procedures increase the intensity of the DAB reaction, not all improve the sensitivity of the detection, as measured by the number of previously undetected cells now visible. Not all these methods are suitable for frozen sections as the morphological detail of the nuclei is impaired.

The silver methenamine enhancement procedure produced the greatest intensity of reaction, but when the NiDABCo and silver enhanced sections were compared with the negative controls, some of this extra labelling was false positivity, probably due to binding of the peroxidase-labelled secondary antibody to Fc receptors on cell membranes. This problem was 
less pronounced for the NiDABCo procedure, but it highlights the fact that negative controls are essential when interpreting results produced by enhancement methods.

Other problems were encountered with the silver enhancement procedures which limit their potential application. Argyrophilic structures, such as reticulin and basal lamina material, may give rise to background staining, and pigments such as melanin may obscure genuine positivity.

The ferric-ferricyanide method resulted in a very clear cut colour change to a pleasing blue-green which, as indicated by the original author, could have a useful application in double-labelling techniques. It might be more profitably applied to paraffin wax sections, however, due to the deleterious effect of the enhancement solution on the nuclear morphology of frozen sections.

Cobalt in Tris- $\mathrm{HCl}$ buffer seems to prevent nuclear material being removed by harmful solutions, as well as intensifying the DAB reaction product and providing nucleation sites for subsequent silver methenamine intensification.

An interesting observation was the difference in background staining produced by the use of cobalt and gold solutions preceding silver methenamine enhancement. In common with immunogold procedures, considerable background of a particulate nature was observed with the gold but not with the cobalt, although the intensity of the reaction was identical. This gives rise to the possibility that immuno-cobalt reagents may produce "cleaner" staining than immunogold-silver staining.

The Tris-cobalt solution can be re-used repeatedly and has applications outside immunocytochemistryfor example, immediately preceding the nuclear counterstain in some enzyme techniques. It seems to conserve the nuclear material with the advantages of lower toxicity and reactivity than mercury or formalin. Its mode of action is unknown. Assuming other factors are optimal, such as section quality, staining technique, and solutions, and that the tissue was frozen and stored correctly, the effect of Tris/cobalt, especially with haematoxylin, is to improve the staining of nuclei to such an extent that nucleoli and chromatin patterns may be discerned more frequently.

Nickel-modified DAB followed by cobalt enhancement (NiDABCo) results in a stable, black deposit which is insoluble in alcohol and xylene. The crispness of the staining means that closely apposed positive cells may be resolved with more success than would be possible with a more diffuse result. The intensity and sensitivity of the staining also greatly facilitates cell counting.

The NiDABCo reaction product is electron dense, making it suitable for electron microscopy, and the black contrasts much better with endogenous pigments, such as haemosiderin and bile, than the goldenbrown of SDAB. The nuclear fast red counterstain enhances the perception of the positivity, permitting low power scanning of sections for occasional positive cells.

The ease with which this technique may be performed, the ability to store the nickel-DAB in aliquots without deterioration, and the potential for labelling a second antigen in the same section with a red, blue or brown reaction product should further recommend this improved method of peroxidase visualisation for frozen sections.

We acknowledge The North of England Cancer Research Campaign for financial support and Mrs Paula McEwen for typing the manuscript.

\section{References}

1 Scopsi L, Larsson L-I. Increased sensitivity in peroxidase immunocytochemistry. Histochemistry 1986;84:221-30.

2 Hsu S-M, Soban E. Color modification of diaminobenzidine precipitation by metallic ions and its application for double immunohistochemistry. J Histochem Cytochem 1982;30: 1079-82.

3 Nemes Z. Intensification of 3-3'diaminobenzidine precipitation using the ferric-ferricyanide reaction, and its application in the double immunoperoxidase technique. Histochemistry 1987;86: 415-9.

4 Rodriguez EM, Yulis R, Peruzzo B, Alvial G, Andrade R. Standardisation of various applications of methacrylate embedding and silver methenamine for light and electron microscopy immunocytochemistry. Histochemistry 1984;81:253-63.

5 Lascano EF, Berria MI. PAP labelling enhancement by osmium tetroxide-potassium ferrocyanide treatment. $J$ Histochem Cytochem 1988;36:697-9.

6 Gallyas F, Görcs T, Merchanthaler I. High grade intensification of the end product of the diaminobenzidine reaction for peroxidase histochemistry. J Histochem Cytochem 1982;30:183-4.

7 Polak JM, van Noorden S. Immunocytochemistry. Bristol: Wright, 1986.

8 Strauss W. Imidazole increases the sensitivity of the cytochemical reaction for peroxidase with diaminobenzidine at a neutral $\mathrm{pH}$. J Histochem Cytochem 1982;30:491-3.

9 Hancock MB. Visualisation of peptide-immunoreactive processes on serotonin-immunoreactive cells using two-colour immunoperoxidase staining. J Histochem Cytochem 1984;32:311-4.

10 Hanker JS, Ambrose WW, James CJ, et al. Facilitated light microscopic cytochemical diagnosis of acute myelogenous leukaemia. Cancer Res 1979;39:1635-9.

Requests for reprints to: Mr M A Green, Department of Pathology, Royal Victoria Infirmary, Queen Victoria Road, Newcastle upon Tyne NE1 4LP, England. 